\title{
Atomic force microscopy characterization of synthetic pyrimidinic oligodeoxynucleotides adsorbed onto an HOPG electrode under applied potential
}

\author{
Ana-Maria Chiorcea Paquim ${ }^{\mathrm{a}}$, Tatiana S. Oretskaya ${ }^{\mathrm{b}}$, Ana Maria Oliveira Brett ${ }^{\mathrm{a}, *}$ \\ ${ }^{a}$ Departamento de Química, Faculdade de Ciências e Tecnologia, Universidade de Coimbra, 3004-535 Coimbra, Portugal \\ b Chemistry Department, Moscow State University, Vorobjovy Gory, 119899 Moscow, Russia \\ Received 13 July 2005 \\ Available online 2 May 2006
}

\begin{abstract}
In order to better understand the adsorption mechanism of nucleic acids at electrode surface, MAC Mode atomic force microscopy (MAC Mode AFM) was used to investigate the adsorption of a 10-base synthetic oligodeoxynucleotide (ODN) with the sequence 5'-CTTTTTCTTT-3' containing only pyrimidinic bases, onto a highly oriented pyrolytic graphite (HOPG) electrode. Free adsorption and adsorption at applied potentials of +0.30 and $+0.65 \mathrm{~V}$ versus AgQRE, were carried out. AFM images in air demonstrated that the molecules adsorb spontaneously on the electrode surface. The ODNs have the tendency to self-assemble from solution onto the solid support in a tight and well-spread two-dimensional lattice covering the entire surface, showing the existence of different molecular conformations and exposing parts of the HOPG surface. The degree of surface coverage and the adsorption pattern were directly dependent on ODN concentration and immobilization procedure. During free adsorption, the hydrophobic interactions of the ODNs with the HOPG represent the main adsorption mechanism. When a positive potential is applied to the HOPG, electrostatic interactions between the positively charged electrode surface and the negatively charged sugar-phosphate backbones of ODNs are predominant. (C) 2006 Elsevier Ltd. All rights reserved.
\end{abstract}

Keywords: Oligodeoxynucleotides; ODN; HOPG; AFM; Atomic force microscopy; DNA-biosensor

\section{Introduction}

DNA electrochemical biosensors are accurate, fast and lowcost devices that are at present under investigation due to their increasingly wider range of medical applications, especially regarding direct monitoring of the hybridization process, the detection of biological warfare agents and specific DNA sequences in human, viral and bacterial nucleic acids in clinical diagnostics [1-6].

A DNA electrochemical biosensor is a transducer (the electrode), with DNA immobilized on its surface. One of the biggest challenges for the development of DNA-biosensor devices for hybridization detection is to understand the hybridization process on the electrode surface $[7,8]$. The hybridization strategy at a DNA electrochemical biosensor consists of three steps: the immobilization of the DNA probe on the electrode, hybridiza-

\footnotetext{
* Corresponding author. Tel.: +351 239 835295; fax: +351 239835295 .

E-mail address: brett@ci.uc.pt (A.M. Oliveira Brett).
}

tion with the complementary strand (target) present in the bulk solution and the signal transduction.

The surface properties of the DNA film immobilized on the electrode surface should be reproducible, stable and selective to complementary DNA sequences. Optimization of the probe layer immobilization procedure is essential for DNA-biosensor technology, since the critical interactions between the DNA molecules and the environment depend directly on the biosensor morphology and structure.

Synthetic oligodeoxynucleotides (ODNs) are ideal chemical recognition elements in a DNA-biosensor for hybridization detection, because they provide an electrochemical signal, the hybridization process is highly sequence-selective and presents minimal cross-hybridization. However, in a DNA-biosensor attention must be paid to the adsorption methodologies of small synthetic ODN probe sequences on the electrode surfaces, in order to achieve probe layer uniformity and functionality.

Atomic force microscopy (AFM) techniques enable direct observation of the topographical properties of non-conductive materials at the molecular level. In recent years a large num- 
ber of studies has been performed using AFM in order to study structural features of immobilized DNA [9]. AFM has been used to investigate DNA morphologies on solid surfaces, DNA condensation for gene therapy and DNA mapping, with applications in cancer research and nanotechnology.

Magnetic AC mode AFM (MAC Mode AFM) permits investigation of DNA molecules weakly bound to the electrode surfaces and was used to study long chain calf-thymus double-stranded DNA (dsDNA) and single-stranded DNA (ssDNA) molecules immobilized onto highly oriented pyrolytic graphite (HOPG) electrode surfaces [10-14]. Therefore, MAC Mode AFM can provide valuable information about electrode surface coverage, orientation and packing of the adsorbed ODN probe layer.

An ODN chain is usually formed by monomeric nucleotide units, each one being composed of three types of chemical component, a phosphate group, a deoxyribose and four different nitrogen bases: adenine $(A)$ and guanine $(\mathrm{G})$, the purine bases, cytosine $(\mathrm{C})$ and thymine $(\mathrm{T})$, the pyrimidine bases [15]. In the present paper a systematic MAC Mode AFM study on a HOPG electrode was carried out at room temperature, concerning the adsorption and conformational changes of a 10-base synthetic ODN which contains only pyrimidinic bases, with the sequence $5^{\prime}$-CTTTTTCTTT-3'. In order to elucidate the mechanism of binding of the short ODN molecules on carbon electrode surfaces, free adsorption and adsorption at applied potentials of +0.30 and $+0.65 \mathrm{~V}$ versus $\mathrm{Ag}$ wire quasi-reference electrode (AgQRE), were carried out.

\section{Experimental}

\subsection{Materials}

The ODN molecules used in this study had the base sequence 5'-CTTTTTCTTT-3' and were synthesized on an Applied Biosystems 380B automated DNA synthesizer (USA) using reagents for ODN chemistry purchased from Fluka (Germany). The purity of the ODN sequences was verified by NMR and HPLC analysis.

Microvolumes were measured using EP-10 and EP-100 Plus Motorized Microliter Pipettes (Rainin Instruments Co. Inc., Woburn, USA). The $\mathrm{pH}$ measurements were carried out with a GLP 21 Crison $\mathrm{pH}$ meter.

The electrolyte used was $\mathrm{pH} 4.50 .1 \mathrm{M}$ acetate buffer solution and was prepared using analytical grade reagents and purified water from a Millipore Milli-Q system (conductivity $<0.1 \mu \mathrm{S} \mathrm{cm}^{-1}$ ). Solutions of different concentrations were obtained by direct dilution of the appropriate volume in acetate buffer.

HOPG grade ZYB, of rectangular shape with $15 \mathrm{~mm} \times$ $15 \mathrm{~mm} \times 2 \mathrm{~mm}$ dimensions, from Advanced Ceramics Co., Germany, was used as substrate. The HOPG was freshly cleaved with adhesive tape prior to each experiment and was imaged by AFM in order to establish its cleanliness.

Voltammetric experiments were carried out in a onecompartment Teflon cell of approximately $12.5 \mathrm{~mm}$ internal diameter holding the HOPG sample, the working electrode, on the base. A Pt wire counter electrode and an Ag wire quasi- reference electrode (AgQRE) were placed in the cell, dipping approximately $5 \mathrm{~mm}$ into the solution. Electrochemical control was done with a PalmSens potentiostat, running with PalmScan version 1.11, from Palm Instruments BV, The Netherlands.

\subsection{Sample preparation}

For ODN samples prepared by free adsorption, $100 \mu \mathrm{L}$ samples of different concentrations of ODN solutions were deposited onto freshly cleaved HOPG surfaces and incubated for $3 \mathrm{~min}$. The excess of ODN was gently cleaned with a jet of Millipore Milli-Q water, and the HOPG with adsorbed ODNs was then dried with nitrogen.

For ODN samples prepared by electrochemical deposition, $500 \mu \mathrm{L}$ of the desired ODN solution was placed in the electrochemical cell holding the HOPG working electrode on the base. Positive potentials of 0.30 and $0.65 \mathrm{~V}$ versus AgQRE, were applied to the electrode for $3 \mathrm{~min}$. The HOPG with adsorbed ODNs was rinsed with a jet of Millipore Milli-Q water and dried with nitrogen.

\subsection{Atomic force microscopy}

AFM was performed with a PicoSPM controlled by a MAC Mode module and interfaced with a PicoScan controller from Molecular Imaging Corp., Tempe, AZ. All the AFM experiments were performed with a CS AFM S scanner with the scan range $6 \mu \mathrm{m}$ in $x-y$ and $2 \mu \mathrm{m}$ in $z$, Molecular Imaging Corp., Silicon type II MAClevers of $225 \mu \mathrm{m}$ length, tip radius of curvature less than $10 \mathrm{~nm}, 2.8 \mathrm{~N} \mathrm{~m}^{-1}$ spring constant, and $60-90 \mathrm{kHz}$ resonant frequencies, Molecular Imaging Corp., were used in MAC Mode AFM. All images (256 samples line ${ }^{-1} \times 256$ lines) were taken at room temperature, with scan rates of 1.0-2.5 lines $\mathrm{s}^{-1}$. The images were processed by flattening in order to remove the background slope, and the contrast and brightness were adjusted. The images were visualized in three dimensions using the Scanning Probe Image Processor, SPIP, version 3.3, Image Metrology ApS, Denmark.

\subsection{Statistics}

Section analysis over DNA molecules and films were performed with PicoScan software version 5.3.1, Molecular Imaging Co. and with Origin version 6.0. from Microcal Software Inc., USA. The mean values of the heights were calculated using 50 measurements over different scanned images. Origin version 6.0 from Microcal Software Inc., USA, was used to calculate standard deviation and to perform the experimental thickness distribution graphs.

\section{Results and discussion}

Atomic force microscopy has been used to reveal the characteristics, density, organization and surface proprieties of immobilized ODNs formed on HOPG using different immobilization procedures. 
The HOPG electrode was modified by free adsorption and by adsorption under applied potential, with thin films of 10-mer synthetic ODNs, sequence $5^{\prime}$-CTTTTTCTTT-3', containing only pyrimidinic bases, and an adsorption time of 3 min was used in all experiments, as described in Section 2.

\subsection{Immobilization of ODN molecules by free adsorption}

Among the various methods that have been reported for the immobilization of nucleic acids, the DNA free adsorption on the electrode surfaces is the easiest, since it does not require any modification of the nucleic acid molecules or of the surface. Consequently, the HOPG electrode modified by thin films of ODN molecules obtained by free adsorption from ODN solution concentrations of $0.1,0.3$ and $0.8 \mu \mathrm{M}$ was investigated.

Free adsorption from a small concentration of $0.1 \mu \mathrm{M}$ showed that the ODN molecules adsorb spontaneously onto HOPG. In the AFM images bright areas appear showing blob-like features that were identified as individual ODN molecules, Fig. 1(A) and (B). The average height and standard deviation of the spherical aggregates was $0.9 \pm 0.3 \mathrm{~nm}$.

The 10-mer ODNs used in this study are very small singlestranded molecules. Assuming that highly dehydrated singlestranded DNA adopts the A-form after drying on the substrate, we estimate that a standard 10-mer ODN molecule has a helix diameter of approximately $2.6 \mathrm{~nm}$, an average axial rise per base of $0.28 \mathrm{~nm}$ and therefore an average length of $2.8 \mathrm{~nm}$, which confers to the 10-mer ODN a spherical appearance. The small single-stranded 10-mer ODN molecules appear in the AFM images as small globular aggregates, with the measured full width at half-maximum height varying between 10 and $30 \mathrm{~nm}$.

The geometric characteristics of the AFM tip are the most important limiting factor in the high resolution imaging of biological materials. Due to the convolution effect of the tip radius, the ODN diameter and length is overestimated in the AFM images. For a spherical AFM tip radius of approximately $10 \mathrm{~nm}$, in contact with a small, well-defined, incompressible and spherical ODN molecule of approximately $2.8 \mathrm{~nm}$ diameter, we expect an ODN measured width of $21.2 \mathrm{~nm}[16,17]$. Nevertheless, since the tip is responsible for such a big contribution to the measured width, it is not possible to distinguish between one or two molecules that condense together.

Additionally, in aqueous solutions, at the $\mathrm{pH} 4.5$ used in this study, which is also the $\mathrm{p} K_{\mathrm{a}}$ of cytidine, many cytosine bases in the ODN chain are protonated, and they are able to form hemiprotonated $\mathrm{CH}^{+}-\mathrm{C}$ and $\mathrm{CH}^{+}-\mathrm{T}$ base-pairs with bases from the same and from other chains [15]. Consequently, singlestranded ODNs have the tendency to fold back on themselves and interact easily with each other, which makes the identification of individual ODN molecules adsorbed on the surface even more difficult.

Height measurements are not limited by the AFM tip radius of curvature and offer a better representation of the ODN diameter; the reported heights for DNA attached to a solid substrate vary from 0.5 to $1.9 \mathrm{~nm}$ for AFM studies in air [18]. Still, height measurements underestimate the ODN helix diameter. The difference between the true DNA height and measured is due to elastic deformations to DNA caused by the AFM tip. Consequently we can conclude that the height of $0.9 \pm 0.3 \mathrm{~nm}$ of the ODN aggregates corresponds to a monolayer of compressed molecules.

The degree of surface coverage increased on increasing the ODN concentration up to $0.3 \mu \mathrm{M}$. The molecules started to organize on the surface in an extended two-dimensional network film, composed of small molecules adsorbed side by side on the surface, Fig. 1(C). The measured thickness of the aggregated fibres forming the network was irregular, and section analyses inside the images permitted the construction of the height distribution graph in Fig. 1(D), which reveals two typical values and indicates that heights from different processes are mixed together. The first height value of $1.1 \pm 0.2 \mathrm{~nm}$ corresponds to a monolayer of molecules. The second value of $1.9 \pm 0.4 \mathrm{~nm}$ is given by the globular aggregates visualized in the images, Fig. 1(D). Since aggregates up to $3 \mathrm{~nm}$ height could be observed in the images, the distribution graph is asymmetric, and the second mean value is strongly influenced by the longer tail of the distribution. Another measure of central tendency of the distribution graph is the mode, defined as the most frequently observed value of the measurements. The mode of the aggregate heights observed in the AFM images was approximately $1.7 \mathrm{~nm}$.

At $0.8 \mu \mathrm{M}$ the ODN adsorbed film became more compact, and more extended areas of the HOPG surface were covered by molecules, Fig. 1(E) and (F). However, the film had many pores - the dark regions in the images - with exposed HOPG surface at the bottom of the pores. The surface of the film was very smooth with a height of approximately $1.0 \pm 0.2 \mathrm{~nm}$, which suggests attractive interaction between the adsorbed ODN molecules that form the monolayer.

\subsection{Immobilization of ODN molecules with applied potential}

The adsorption process on the HOPG surface can be controlled by applying a positive potential to the electrode surface, because the negatively charged phosphate groups of the ODN molecules are electrostatically attracted by a positively charged HOPG electrode surface.

Most electrochemical DNA-biosensors are based on the determination of purine oxidation peaks, principally the guanine peak, to monitor the hybridization of ODN probe molecules immobilized on the electrode with the ODN complementary sequences in solution $[1,2,7,8]$.

Electrochemical studies at glassy carbon electrodes showed in detail the oxidation mechanism of the DNA bases. Guanine and adenine were reported to be the easiest oxidised bases in DNA. The guanine oxidation peak appears at approximately $+0.60 \mathrm{~V}$ versus $\mathrm{AgQRE}$, confirming that guanine has the lowest oxidation potential of all DNA bases, and the oxidation of adenine appears at $+0.86 \mathrm{~V}$. Thymine and cytosine are more difficult to detect when compared with guanine and adenine, the oxidation peak of thymine appearing at $+1.06 \mathrm{~V}$, and of cytosine at $+1.21 \mathrm{~V}$ versus AgQRE [19-22] 

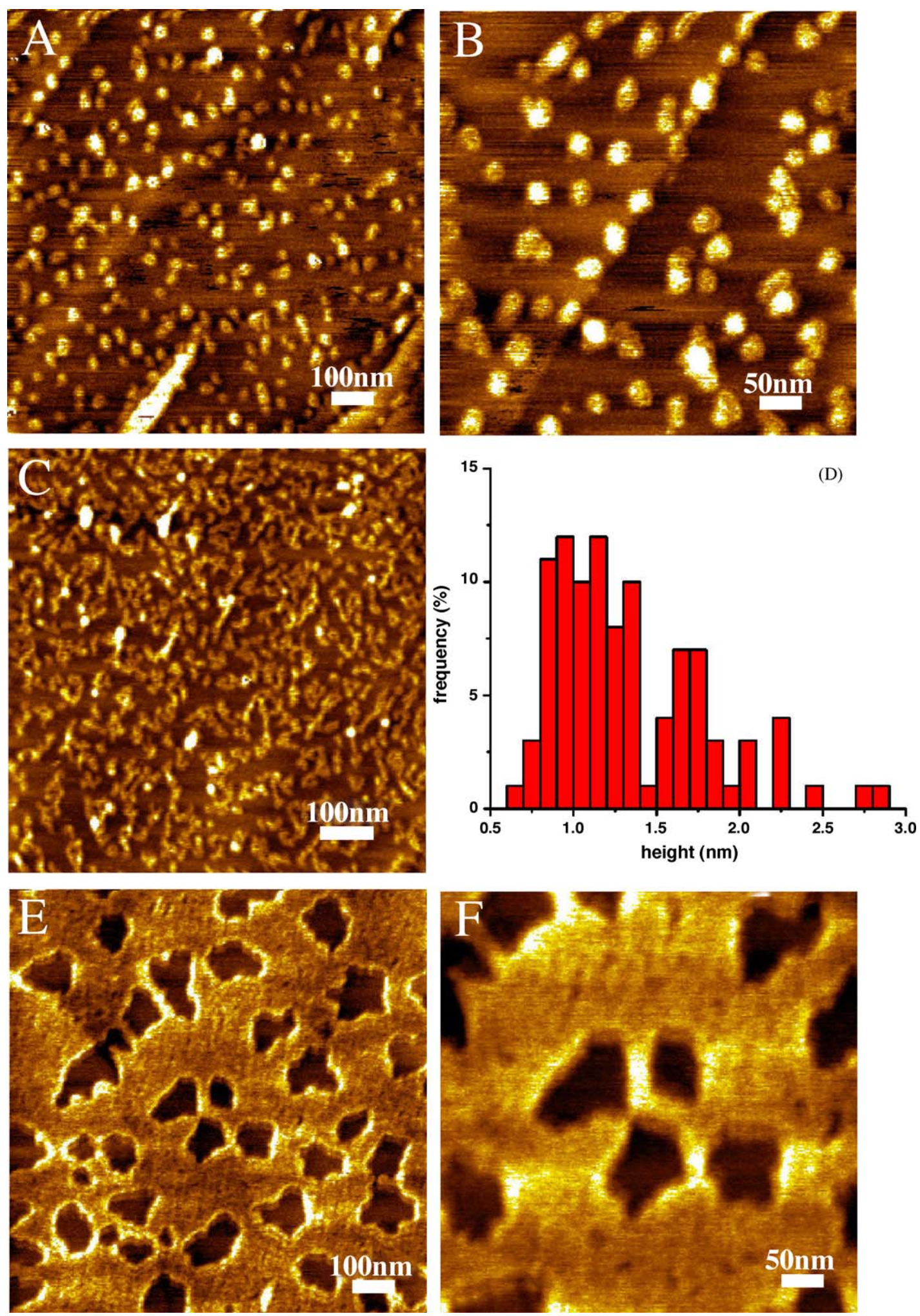

Fig. 1. MAC Mode AFM topographical images in air of ODN molecules, immobilized on the HOPG surface by free adsorption during 3 min, from a (A and B) $0.1 \mu \mathrm{M}(\mathrm{C}) 0.3 \mu \mathrm{M}$ and (E and F) $0.8 \mu \mathrm{M}$ ODN in $\mathrm{pH} 4.50 .1 \mathrm{M}$ acetate buffer solution. (D) Histogram of the ODN layer thickness generated with the values measured by section analysis inside the images.

Two different positive potentials, of +0.30 and $+0.65 \mathrm{~V}$ versus AgQRE, were chosen to study the effect of electrochemically assisted adsorption of ODN molecules on the HOPG electrode surface.
The potential of $+0.30 \mathrm{~V}$ is not sufficiently high to oxidize any of the DNA bases, while the potential of $+0.65 \mathrm{~V}$ is sufficiently high to oxidize the guanine bases in the DNA. However, the particular choice of an ODN sequence containing only pyrimidinic 

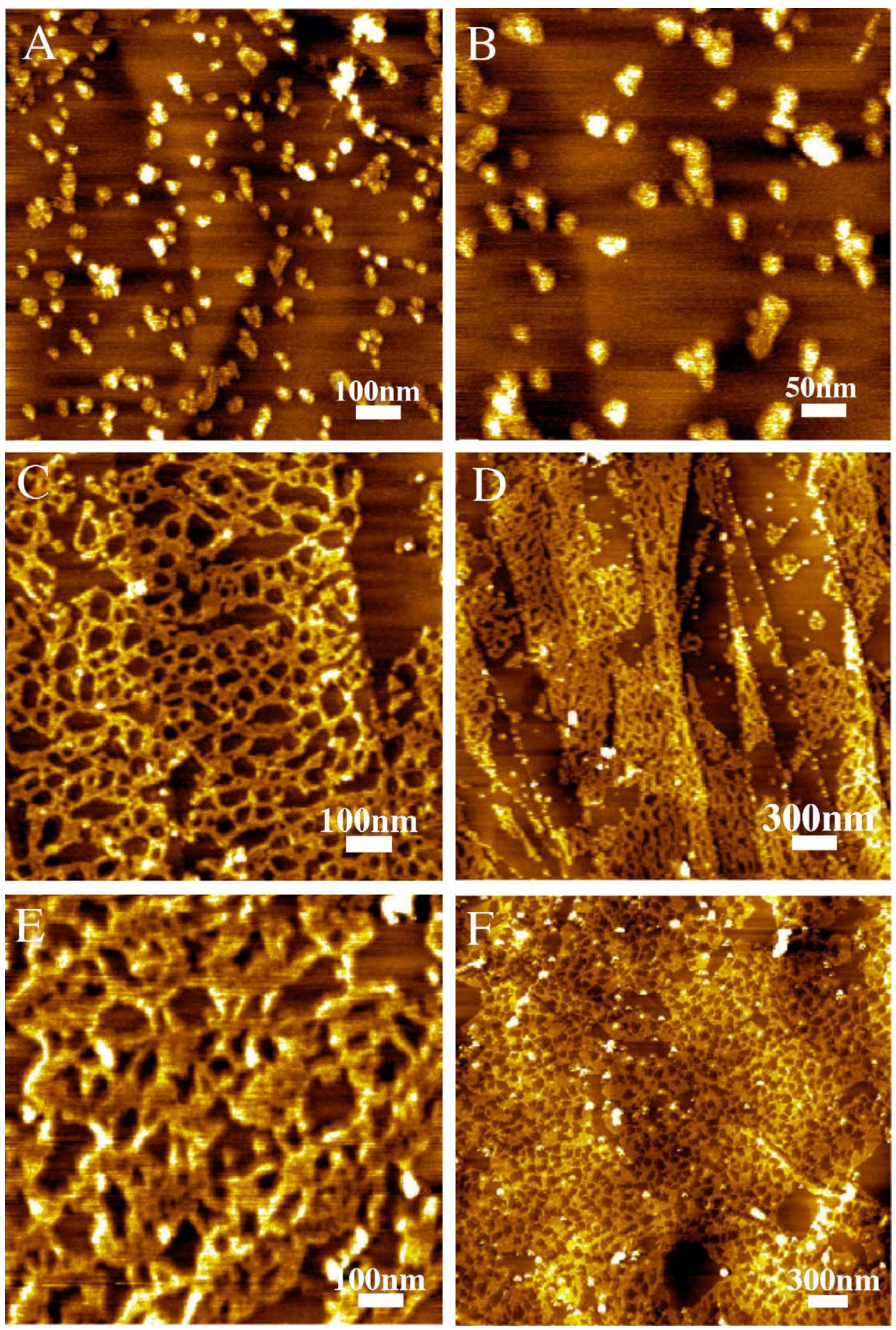

Fig. 2. MAC Mode AFM topographical images in air of ODN molecules, immobilized on the HOPG surface by adsorption during 3 min, at $+0.30 \mathrm{~V}$ vs. AgQRE applied potential, from a (A and B) $0.1 \mu \mathrm{M}(\mathrm{C}$ and D) $0.3 \mu \mathrm{M}$ and $(\mathrm{E}$ and $\mathrm{F}) 0.8 \mu \mathrm{M} \mathrm{ODN}$ in pH $4.50 .1 \mathrm{M}$ acetate buffer solution. 
bases allows the study of the ODN probe layer adsorption at higher potentials, without causing the oxidation of the bases in the ODN sequence.

The HOPG electrode was immersed in ODN solutions of different concentrations, under the conditions described in Section 2, and the surface morphology and stability of the ODN adsorbed layer were analyzed by MAC mode AFM in air. The surface coverage with increasing ODN concentration was monitored, the characteristics of the ODN film depending strongly on the solution concentration.

\subsubsection{Adsorption at $+0.30 \mathrm{~V}$ applied potential}

The effect of the $+0.30 \mathrm{~V}$ versus AgQRE, applied potential on the adsorption and stability of the ODN layer onto HOPG, was determined using concentrations of $0.1,0.3$ and $0.8 \mu \mathrm{M}$ ODN.

Typical AFM images of the ODN layer adsorbed from a concentration of $0.1 \mu \mathrm{M}$ are presented in Fig. 2(A) and (B). The molecule adsorbed as an agglomeration of small aggregates with the height of $1.1 \pm 0.4 \mathrm{~nm}$. The degree of HOPG surface coverage with ODN molecules was similar to that obtained by free adsorption. However, the greater height of the molecules, when compared with the measured height of freely adsorbed ODNs, suggests that the ODNs were more stable on the surface due to electrostatic interaction with the HOPG surface. Consequently, the molecules were less compressed by the AFM tip, while scanning the sample.

At $0.3 \mu \mathrm{M}$, an ODN network film covering the HOPG electrode surface was imaged, Fig. 2(C). The ODN molecules demonstrated predisposition to condense together in extensive thin lattices covering big areas of the substrate. Between neighbouring thin lattices, large zones of the HOPG were not covered by the ODN film, as shown in the large-scale image from Fig. 2(D). The measured thickness of the film was $1.3 \pm 0.2 \mathrm{~nm}$, and the stability of the ODN layer was also improved due to electrostatic interaction with the positively charged HOPG electrode surface.

Raising the ODN concentration to $0.8 \mu \mathrm{M}$ resulted in an increase of the degree of surface coverage. The AFM images in air also showed a uniform self-assembled network with thicker looped arms, Fig. 2(E). The HOPG electrode became almost entirely covered by molecules, Fig. 2(F), and the thickness of the monolayer film was $1.1 \pm 0.2 \mathrm{~nm}$.
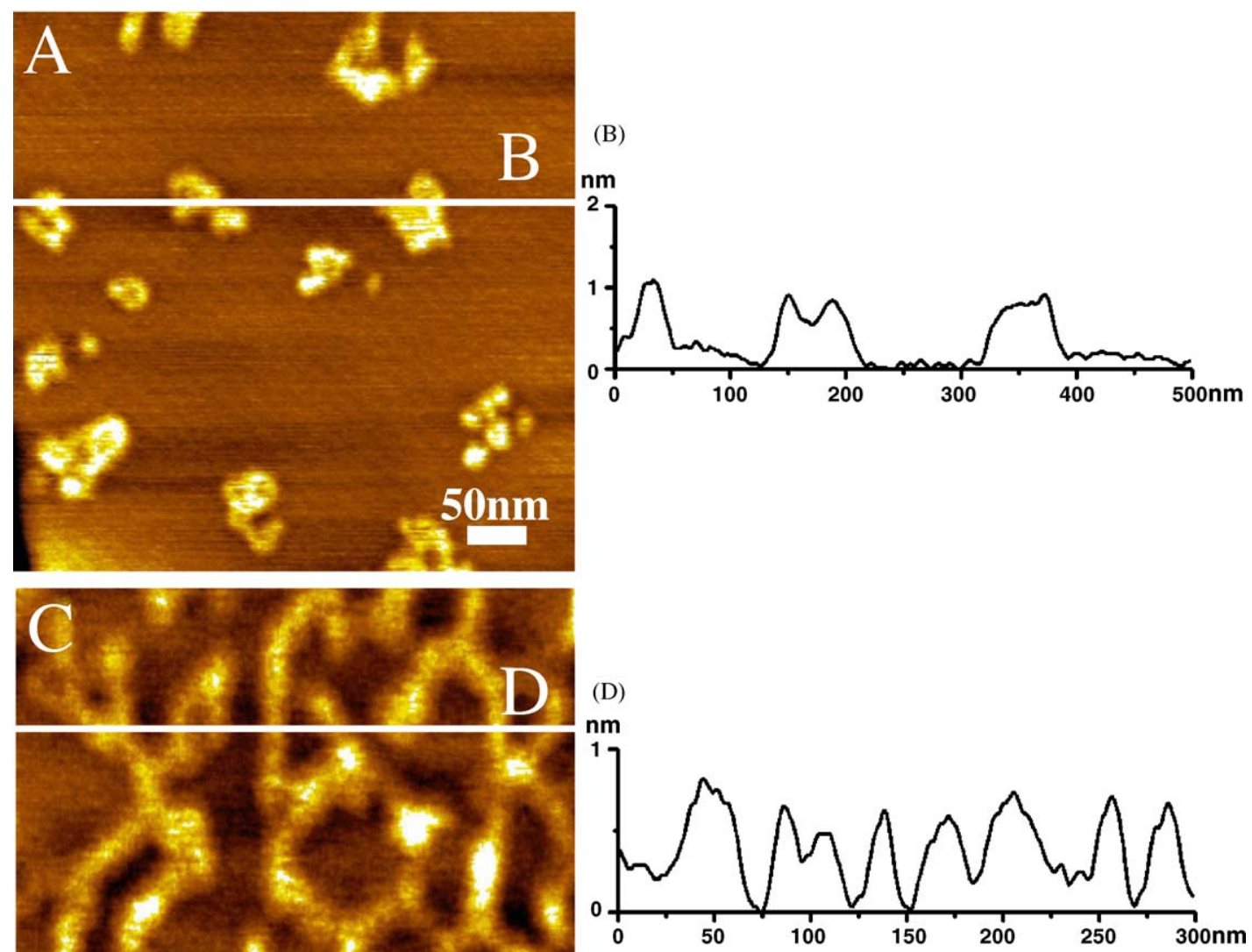

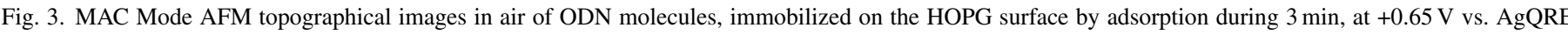

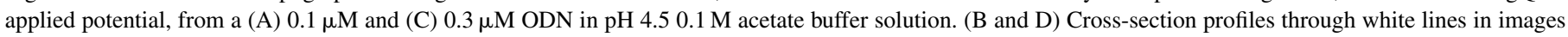
(A) and (C). 


\subsubsection{Adsorption at $+0.65 \mathrm{~V}$ applied potential}

The experiments with $+0.65 \mathrm{~V}$ versus $\mathrm{AgQRE}$, applied potential were performed also for three different solution concentrations, and the ODN adsorbed layers have been observed.

At an ODN concentration of $0.1 \mu \mathrm{M}$, the HOPG surface was sparsely covered by ODN aggregates with average height of $0.9 \pm 0.3 \mathrm{~nm}$, Fig. 3(A) and (B), consistent with a monolayer of
ODN molecules. As predicted, the ODN molecules adsorbed at $+0.65 \mathrm{~V}$ also showed higher stability on the surface compared with ODNs immobilized by free adsorption.

When the ODN concentration was increased to $0.3 \mu \mathrm{M}$, the HOPG electrode became homogeneously covered by a thin twodimensional network film formed by linear strands and covering the large domains on the HOPG surface terraces, Fig. 3(C).
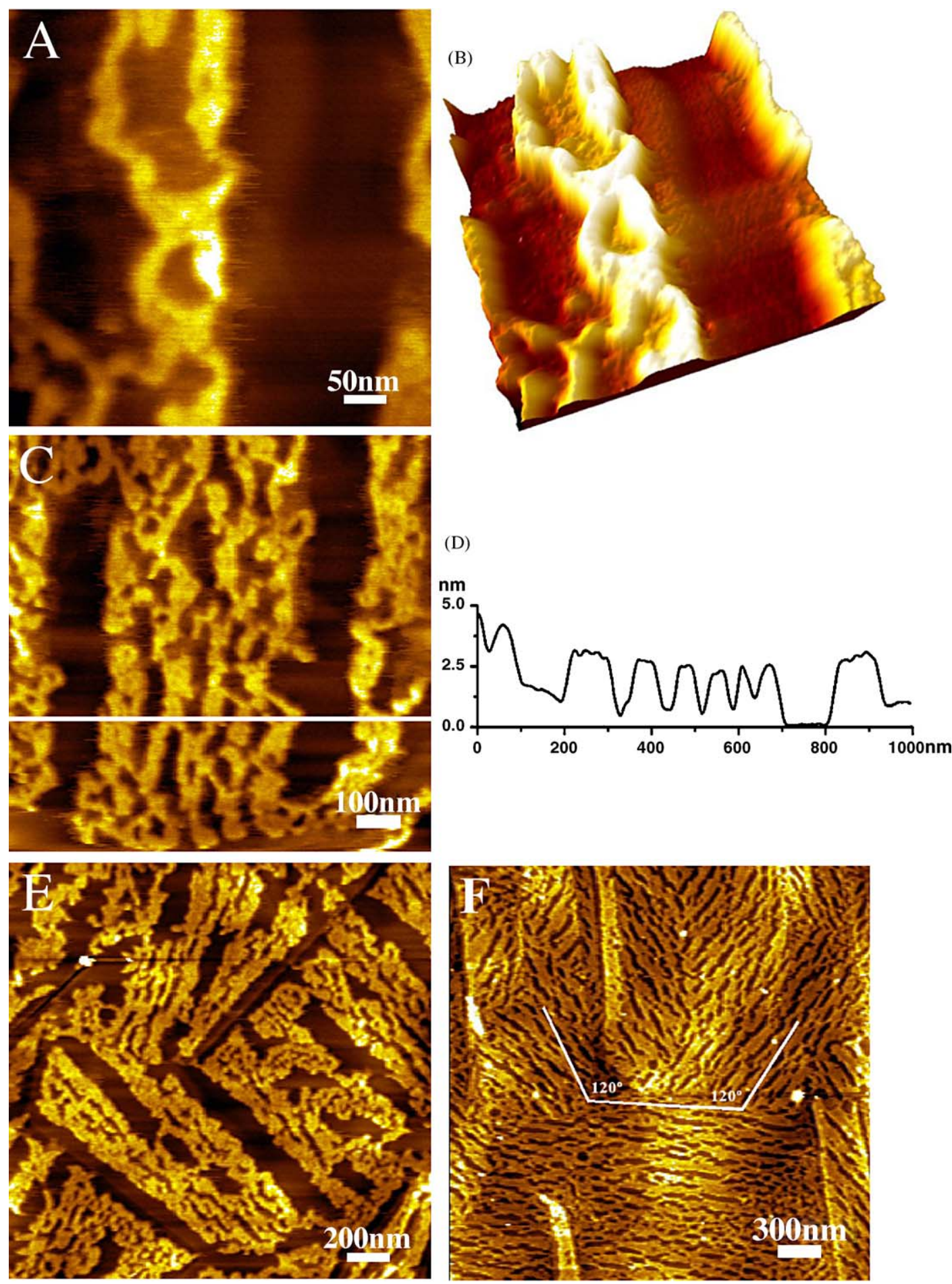

Fig. 4. MAC Mode AFM topographical images in air of ODN molecules, immobilized on the HOPG surface by adsorption during $3 \mathrm{~min}$, at $+0.65 \mathrm{~V}$ vs. AgQRE applied potential, from $0.8 \mu \mathrm{M}$ ODN in pH 4.5 0.1 M acetate buffer solution. (B) Three-dimensional representation of image (A). (D) Cross-section profile through white line in image $(\mathrm{C})$. 
Variable angles between the network strands were observed. The network height was $0.8 \pm 0.2 \mathrm{~nm}$ as measured by section analyses inside the AFM images, Fig. 3(D). DNA networks formed by linear strands on the HOPG electrode surface were also observed for long chain calf-thymus ssDNA adsorbed under applied potential [13].

The height of the molecules and monolayer films obtained at $+0.65 \mathrm{~V}$ was smaller than the height of the ODNs adsorbed at $+0.30 \mathrm{~V}$. The higher applied potential of $+0.65 \mathrm{~V}$ modifies the conformation of the ODN molecules on the electrode surface. The positively charged HOPG substrate exerts a strong electrostatic attraction on the negatively charged ODNs, in such a way that all the phosphate groups become attached to the surface; the single-stranded ODN helical configuration becomes stretched on the HOPG surface, and consequently leads to a decrease of the molecule diameter as measured by AFM.At a high ODN concentration of $0.8 \mu \mathrm{M}$, the applied potential had a strong impact on the self-assembling properties of the ODN molecules containing pyridine bases. The small ODN molecules tended to adsorb close to each other, in very close packed chains, leading to the appearance of large polymeric-like assemblies, Fig. 4(A) and (B). The narrow polymeric structures formed larger assemblies on the surface, Fig. 4(C), bright bands. The polymeric bands produced by the ODN molecules presented ordered packing on the HOPG surface, remaining parallel to each other over extended domain lattices. The measured thickness of ODN lattices was $2.0 \pm 0.5 \mathrm{~nm}$, Fig. 4(D), which suggests that more than one monolayer of ODN molecules condensed together. The ODNs interact easily with each other, due to the stretching of the ODN sequence on the surface that destabilized the base stacking of the molecules.

HOPG is a polycrystalline material, consisting of individual crystalline domains which are rotated against each other. The cleaving process of the HOPG electrode with adhesive tape reveals many different atomically flat terraces on the basal plane of graphite. For instance, the topmost monolayer of the HOPG surface may be torn apart during the cleaving process, leaving rectangular holes with the depth of a mono- or multiple-layers on the graphite surface.

Large-scale AFM images revealed changes of orientation of the ODN adsorbed layer near HOPG surface defects, and permitted the visualization of the ODN molecules adsorbed on various HOPG substrate terraces, Fig. 4(E) and (F). On flat HOPG terraces, the adjacent domains adsorbed were oriented mainly along three directions, at $60^{\circ}$ and $120^{\circ}$ to each other, dictated by the three-fold symmetry of the graphite substrate and by the interactions of the ODN chains with the substrate. On other HOPG terraces, ODN lattice domains running along directions oriented at different angles were also observed.

The formation of these elongated lattices, running in the same direction as the underlying graphite surface were observed consistently in various independent experiments, run in the same experimental conditions.

As observed in the images, the molecular polymeric chains were not oriented at a fixed angle relative to the direction of the step edges of the substrate, indicating that the orientation of the ODN parallel polymeric structures was not induced by particular surface defects at HOPG step edges, but induced by the perfectly flat HOPG terraces.The preferential selfassembling of the ODN molecules at $+0.65 \mathrm{~V}$, on the basal plane of the HOPG surface, in such a manner that the orientation of the adsorbed ODN layer resembles the crystalline orientation of the substrate is reminiscent of epitaxy growth. DNA bases are also known to form highly ordered monolayers on the basal plane of HOPG substrate, observed commonly with scanning tunneling microscopy (STM) [23]. This suggests that, after destabilization of the ODN structure due to the strong interaction of the negatively charged phosphate groups with the positive HOPG electrode, the ODN bases adsorb easily on the HOPG surface, stabilizing the adsorbates.

\section{Conclusions}

As observed in the AFM images in air, ODN molecules adsorb spontaneously and rapidly on HOPG electrode surfaces. The degree of surface coverage, the molecular conformation, the layer arrangement and the adsorption pattern of the ODN molecules were strongly affected by the ODN concentration, immobilization procedure and the specific interaction of DNA bases with the HOPG substrate.

At small ODN concentrations, the ODNs appear as small globular aggregates; increasing the concentration of the ODN solution the molecules self-assemble in tight and well-spread two-dimensional lattices covering the entire surface and exposing parts of the HOPG electrode.

During free adsorption, the hydrophobic interactions of the ODN molecules with the HOPG surface represent the main adsorption mechanism, although other effects such as electrostatic and Van der Waals interactions also contribute to the adsorption process. When a positive potential was applied to the HOPG surface, electrostatic interactions with the HOPG electrode are predominant, followed by the interaction of the bases with the surface.

The applied potential did not increase the HOPG surface coverage with pyrimidinic ODNs, but modified the ODN adsorption pattern on the surface significantly. The robustness and stability of the adsorbed molecules with respect to mechanical stress increased, due to electrostatic interaction between the positively charged electrode surface and the negatively charged sugar-phosphate backbone.

\section{Acknowledgments}

Financial support from Fundação para a Ciência Tecnologia (FCT), Post-Doc Grant SFRH/BPD/14425/2003 (A.-M. Chiorcea Paquim), POCTI (co-financed by the European Community Fund FEDER), ICEMS (Research Unit 103), and European Project HPRN-CT-2002-00186 are gratefully acknowledged.

\section{References}

[1] A.M. Oliveira Brett, in: L. Gorton (Ed.), Comprehensive Analytical Chemistry, Biosensors and Modern Specific Analytical Techniques, vol. 44, Elsevier, 2004, p. 179 (Chapter 8). 
[2] A.M. Oliveira Brett, S.H.P. Serrano, J.A.P. Piedade, in: R.G. Compton, G. Hancock (Eds.), Comprehensive Chemical Kinetics, Applications of Kinetic Modeling, vol. 37, Elsevier, Oxford, UK, 1999, p. 91 (Chapter 3).

[3] E. Palecek, M. Fojta, F. Jelen, Bioelectrochemistry 56 (2002) 85.

[4] E. Palecek, M. Fojta, M. Tomschik, J. Wang, Biosens. Bioelectron. 13 (1998) 621.

[5] J. Wang, X. Cai, G. Rivas, H. Shiraishi, P.A.M. Farias, N. Dontha, Anal. Chem. 68 (1996) 2629.

[6] G. Marrazza, I. Chianella, M. Mascini, Biosens. Bioelectron. 14 (1999) 43.

[7] A.-M. Chiorcea Paquim, V.C. Diculescu, T.S. Oretskaya, A.M. Oliveira Brett, Biosens. Bioelectron. 20 (2004) 933.

[8] A.M. Oliveira Brett, A.-M. Chiorcea Paquim, V. Diculescu, T.S. Oretskaya, Bioelectrochemistry 67 (2005) 181.

[9] H.G. Hansma, Annu. Rev. Phys. Chem. 52 (2001) 71.

[10] A.M. Oliveira Brett, A.-M. Chiorcea Paquim, Electrochem. Commun. 5 (2003) 178.

[11] A.M. Oliveira Brett, A.-M. Chiorcea Paquim, Langmuir 19 (2003) 3830.

[12] A.M. Oliveira Brett, A.-M. Chiorcea Paquim, Bioelectrochemistry 63 (2004) 229.
[13] A.M. Oliveira Brett, A.-M. Chiorcea Paquim, Bioelectrochemistry 66 (2005) 117.

[14] S. Tanaka, Y. Maeda, L.T. Cai, H. Tabata, T. Kawai, Appl. Phys. 40 (2001) 4217.

[15] W. Saenger, in: Ch.R Cantor (Ed.), Springer Advanced Texts in Chemistry, Springer-Verlag, New York, 1984.

[16] F. Zenhausern, M. Adrian, R. Emch, M. Taborelli, M. Jobin, P. Descouts, Ultramicroscopy 42-44 (1992) 1168.

[17] A.-M. Chiorcea Paquim, T.S. Oretskaya, A.M. Oliveira Brett, Biophys. Chem. 121 (2006) 131.

[18] H.G. Hansma, I. Revenko, K. Kim, D.E. Laney, Nucleic Acids Res. 24 (1996) 713.

[19] A.M. Oliveira-Brett, J.A.P. Piedade, L.A. Silva, V.C. Diculescu, Anal. Biochem. 332 (2004) 321.

[20] A.M. Oliveira Brett, F.-M. Matysik, J. Electroanal. Chem. 429 (1997) 95.

[21] A.M. Oliveira Brett, F.-M. Matysik, Bioelectrochem. Bioener. 42 (1997) 111.

[22] A.M. Oliveira Brett, L.A. Silva, C.M.A. Brett, Langmuir 18 (2002) 2326.

[23] S.J. Sowerby, W.M. Heckl, Origins Life Evol. B. 28 (1998) 283 